\title{
Wolff-Parkinson-White Syndrome
}

National Cancer Institute

\section{Source}

National Cancer Institute. Wolff-Parkinson-White Syndrome. NCI Thesaurus. Code C35132.

A cardiac conduction disorder characterized by an electrocardiog raphic finding of ventricular pre-excitation, which is a short PR interval and a long QRS interval with a delta wave. Most individuals are asymptomatic; however they can experience periods of palpitations, shortness of breath or syncope during tachycardic episodes. 